\title{
KRAS Mutation in Metastatic Pancreatic Ductal Adenocarcinoma: Results of a Multicenter Phase II Study Evaluating Efficacy of Cetuximab plus Gemcitabine/Oxaliplatin (GEMOXCET) in First-Line Therapy
}

\author{
F. Kullmann ${ }^{a} \quad$ A. Hartmann ${ }^{b}$ R. Stöhr ${ }^{b}$ H. Messmann ${ }^{c} \quad$ M.M. Dollinger ${ }^{d}$

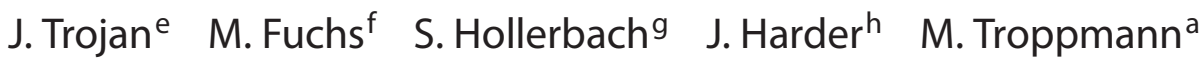 \\ A. Kutscheidt ${ }^{i}$ E. Endlicher ${ }^{a}$ \\ a University of Regensburg, Regensburg, ${ }^{b}$ University of Erlangen, Erlangen, ' $A$ Academic Teaching Hospital,

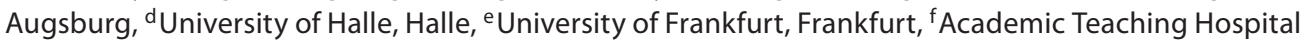 \\ Munich-Bogenhausen, Munich, ${ }^{9}$ Academic Teaching Hospital, Celle, hepartment of Medicine Il, \\ University Medical Center, University of Freiburg, Freiburg, and 'WISP Research Institute, Langenfeld, Germany
}

\section{Key Words}

Pancreatic cancer · Chemotherapy · KRAS mutation •

Cetuximab $\cdot$ Gemcitabine $\cdot$ Oxaliplatin

\begin{abstract}
Background: Genetic alterations within the epidermal growth factor receptor (EGFR) pathway, including KRAS mutations, have been demonstrated to be associated with response to EGFR inhibitors like cetuximab in colorectal cancers. Mutations in the KRAS gene have been found in $70-$ $90 \%$ of pancreatic cancers. Unfortunately, the addition of cetuximab to chemotherapy did not increase response or survival in patients with advanced pancreatic cancer in phase II and phase III studies. The aim of this study was to evaluate the relationship between KRAS mutations and response or survival in patients with metastatic pancreatic cancer treated with cetuximab plus chemotherapy. Methods: Within a multicenter phase II trial, 64 patients with metastatic pancreatic cancer were treated with cetuximab in
\end{abstract}

combination with gemcitabine and oxaliplatin until disease progression. Analyses of the EGFR pathway, including KRAS mutations, could be performed in 25 patients. Analyses were carried out following microdissection of the tumor. Results: Fourteen (56\%) of the 25 patients examined harbored a point mutation in codon 12 of the KRAS gene. No differences between the groups were noted in median progression-free survival (104 days in KRAS wild-type patients vs. 118 days in patients with KRAS mutations). Overall survival was longer in wild-type patients compared to patients with KRAS mutations ( 263 vs. 162 days), but the difference did not reach statistical significance. A further analysis of our clinical phase II trial showed that the presence of a rash was significantly correlated with overall survival. Conclusions: KRAS mutation in codon 12 may be associated with reduced survival compared to KRAS wild type. The role of KRAS mutations for cetuximab therapy in pancreatic cancer warrants further investigation in larger trials to exclude an epiphenomenon. Furthermore, the development of a rash is indicative of clinical benefit.

\section{KARGER}

Fax +4161306 1234 E-Mail karger@karger.ch www.karger.com

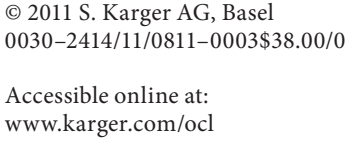

F. Kullmann, MD

Kliniken Nordoberpfalz AG - Klinikum Weiden

Söllnerstrasse 16

DE-93637 Weiden (Germany)

Tel. +49961303 3102, E-Mail frank.kullmann@kliniken-nordoberpfalz.ag 


\section{Introduction}

The prognosis for patients with pancreatic adenocarcinoma is still dismal. Since 1997, gemcitabine has been accepted as the standard palliative chemotherapy for advanced pancreatic cancer, with a median survival of 6 months [1]. Many trials have been conducted evaluating various combination protocols. Meta-analysis of randomized trials revealed that pancreatic cancer patients with good performance status appear to benefit from gemcitabine-based cytotoxic combinations [2]. Nevertheless, data are conflicting as shown in a recently published study by Colucci et al. [3]. In this randomized phase III trial, the addition of weekly cisplatin to gemcitabine failed to demonstrate any improvement as firstline treatment of advanced pancreatic cancer.

Therefore, there is a strong need for effective treatment modalities. With respect to molecular biology, the epidermal growth factor receptor (EGFR) has been shown to play an important role in carcinogenesis of pancreatic cancer $[4,5]$. Moore et al. [6] reported a statistically significant overall survival benefit of 0.33 months for erlotinib, an oral reversible inhibitor of EGFR tyrosine kinase, in combination with gemcitabine compared with gemcitabine alone for first-line therapy in patients with advanced pancreatic cancer. Cetuximab, a monoclonal antibody targeting the EGFR, also resulted in promising activity in preclinical and early clinical trials $[7,8]$. Thus, we recently conducted a phase II study to assess the efficacy and safety of cetuximab plus the combination of gemcitabine/oxaliplatin in metastatic pancreatic cancer [9]. In 64 patients with metastatic pancreatic carcinoma the addition of cetuximab to the combination of gemcitabine and oxaliplatin was well tolerated and exhibited a high response rate (33\%). The median time to progression-free survival was 3.9 months and overall survival was 7.1 months.

The aim of this study was to evaluate the relationship between KRAS mutations and response or survival in patients with metastatic pancreatic cancer treated with cetuximab plus chemotherapy, based on tumor samples available from the recently conducted study [9].

\section{Methods}

This study was conducted as part of a multicenter phase II trial of 64 patients with metastatic pancreatic cancer [9]. Eligible patients were treated with cetuximab in combination with gemcitabine and oxaliplatin until disease progression. All participants for mutation analysis gave written informed consent. Ethical approval for the retrospective use of paraffin material for the

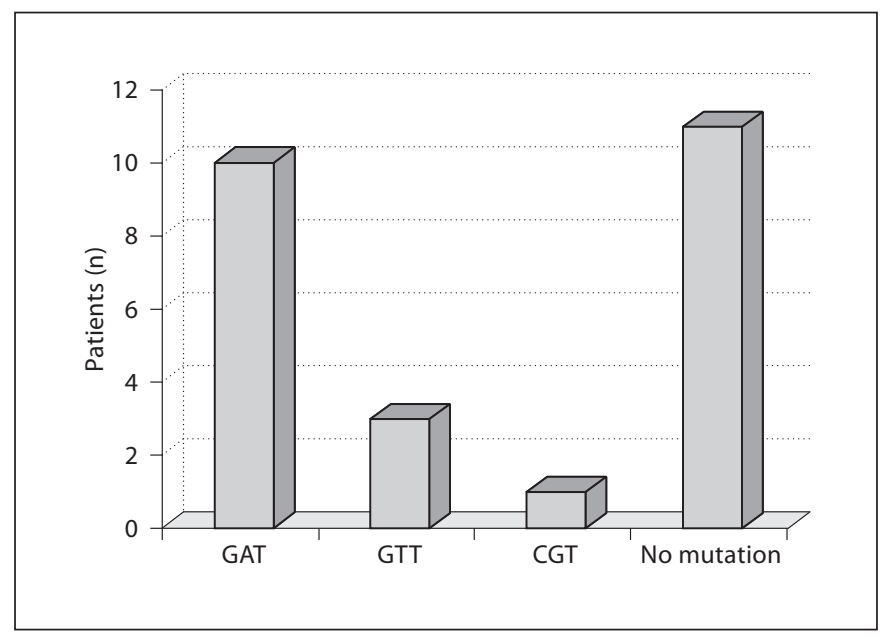

Fig. 1. Distribution of KRAS mutations.

study was given by the ethical committee at the University of Regensburg.

Tumor material was available for the study from a total of 25/64 (39\%) patients, of whom 10 were from Regensburg, 4 from Augsburg, 4 from Halle, 4 from Frankfurt and 3 from Munich. For the remaining cases, there were either no paraffin blocks available or there was not sufficient tumor tissue in the block for molecular analysis. Analyses of KRAS mutations were performed at the Institute of Pathology in Erlangen. All pathologic specimens were cut from formalin-fixed paraffin-embedded tumor blocks, and the HE-stained section was reviewed by one pathologist (A.H.). Tumor cells were manually microdissected from paraffin sections of $10-\mu \mathrm{m}$ thickness under an inverted microscope to obtain a tumor cell population of $70 \%$.

DNA was isolated using the QIAamp ${ }^{\circledR}$ DNA FFPE Tissue Kit (QIAGEN, Hilden, Germany). Quantity and quality of the DNA were controlled using a spectral photometer (NanoDrop ${ }^{\circledR}$, peQLab, Erlangen, Germany). Further details were described previously [10].

Statistical analysis was performed using the SPlus software (Insightful Corp., Seattle, Wash., USA). Comparisons of KaplanMeier curves were based on the two-sided log-rank test.

\section{Results}

Baseline characteristics of the evaluable patient population are shown in table 1 . Fourteen (56\%) of the 25 pancreatic adenocarcinomas examined harbored a point mutation in the KRAS gene. All KRAS mutations occurred at codon 12. The distribution of different KRAS mutations is shown in figure 1 . The most frequently observed point mutation at codon 12 was GAT Gly12Asp followed by GTT Gly12 Val. 
Fig. 2. Overall survival depending on KRAS mutation.

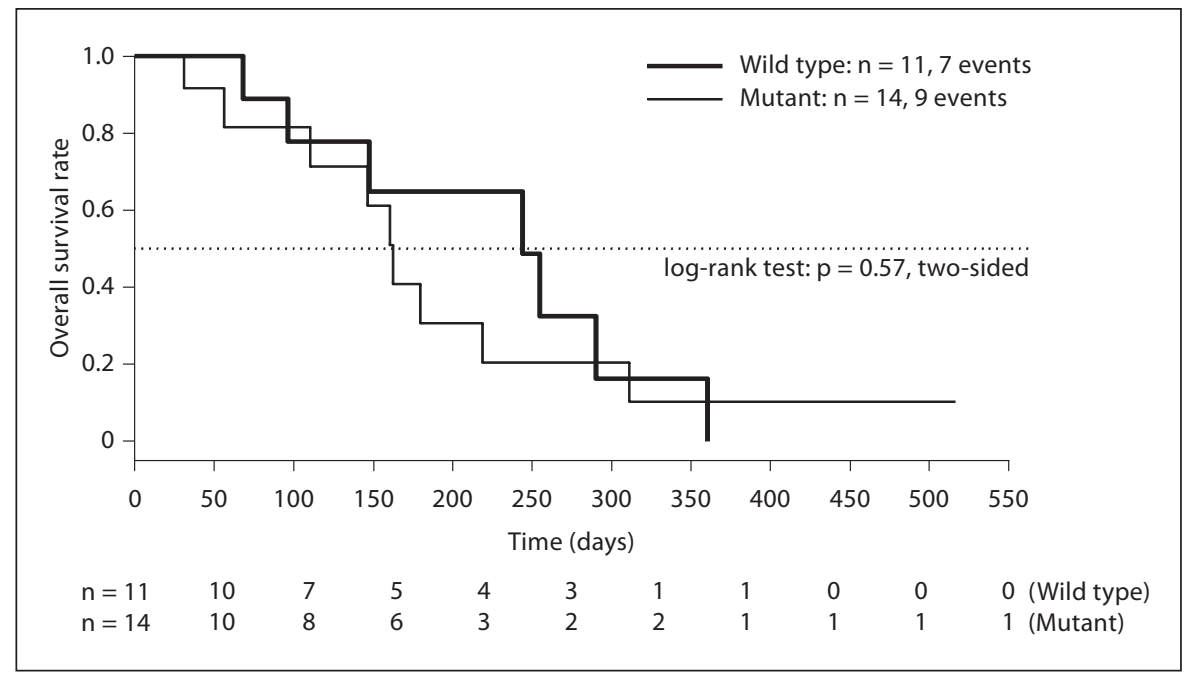

Table 1. Baseline characteristics

\begin{tabular}{lcc}
\hline & $\begin{array}{l}\text { Investigated } \\
\text { subgroup } \\
\text { of patients }\end{array}$ & $\begin{array}{c}\text { Whole study } \\
\text { population }\end{array}$ \\
\hline $\begin{array}{lc}\text { Patients, } \mathrm{n} \\
\text { Mean age (range), years }\end{array}$ & 25 & 62 \\
Male/female, $\mathrm{n}$ & $61.4(37-72)$ & $64.5(31-78)$ \\
Performance status, $\mathrm{n}$ & $19 / 6$ & $41 / 21$ \\
$\quad$ & $2(10 \%)$ & $6(12 \%)$ \\
80 & $9(43 \%)$ & $13(25 \%)$ \\
$\quad 10-100$ & $10(28 \%)$ & $33(63 \%)$ \\
Distant metastasis, $\mathrm{n}$ & $1(4 \%)$ & $8(1 \%)$ \\
$\quad$ Lymph nodes (abdominal/pelvic) & $23(92 \%)$ & $58(93 \%)$ \\
$\quad$ Liver & $4(16 \%)$ & $8(1 \%)$ \\
$\quad$ Lung & $9(36 \%)$ & $22(3 \%)$ \\
$\quad$ Other & 0 & 1 \\
Adjuvant treatment (before start of trial), $\mathrm{n}$ & & \\
\hline
\end{tabular}

The presence of KRAS point mutations at codon 12 adversely influenced median survival time [162 vs. 263 days, KRAS mutation (+ vs. -), fig. 2], but the difference did not reach statistical significance $(\mathrm{p}=0.57$; HR 1.33; 95\% CI 0.49-3.61). The overall survival rate after 6 months was $65 \%$ (95\% CI 39-100\%) in the wild-type group versus $31 \%$ (95\% CI 12-78\%) in the group with KRAS codon 12 mutations.

Median progression-free survival was 104 days in the wild-type group compared to 118 days within the group with KRAS mutation ( $\mathrm{p}=0.63$; HR 0.79). In the wildtype group, progression-free survival after 6 months was $20 \%$ (95\% CI 6-69\%) and 22\% (95\% CI 7-75\%) in the group with KRAS codon 12 mutation. Correlation of the
Table 2. Correlation of KRAS status with response (\%)

\begin{tabular}{|c|c|c|c|}
\hline & $\begin{array}{l}\text { KRAS wild type } \\
(\mathrm{n}=11)\end{array}$ & $\begin{array}{l}\text { KRAS mutation } \\
(\mathrm{n}=13)\end{array}$ & $\begin{array}{l}\text { Total } \\
(\mathrm{n}=24)\end{array}$ \\
\hline Complete response & - & - & - \\
\hline Partial response & $2(18)$ & $5(38)$ & $7(28)$ \\
\hline Stable disease & $4(36)$ & $1(8)$ & $5(20)$ \\
\hline Progressive disease & $5(45)$ & $7(54)$ & $12(50)$ \\
\hline
\end{tabular}

KRAS status with response is shown in table 2. A further analysis of our initial clinical phase II trial showed that the presence of a rash ( $\geq$ grade 2 ) was significantly correlated with overall survival (fig. 3). Sixty-one patients could be included in this analysis. Skin toxicity was usually mild to moderate (grade 1 or 2) in 43 (70\%) patients; only $6(10 \%)$ developed a grade 3 acne-like rash. Patients developing any grade of rash had a significantly longer median overall survival of 237 days compared to 148 days for patients with no rash ( $\mathrm{p}=0.014$; HR 2.37; 95\% CI 1.16-4.85).

\section{Discussion}

Systemic chemotherapy has so far failed to demonstrate sufficient impact on the survival in patients with advanced pancreatic cancer. The EGFR pathway is a rational molecular target for an alternative therapeutic approach. After promising preclinical and clinical trials [7, 8] findings of further phase II and III studies suggest that 


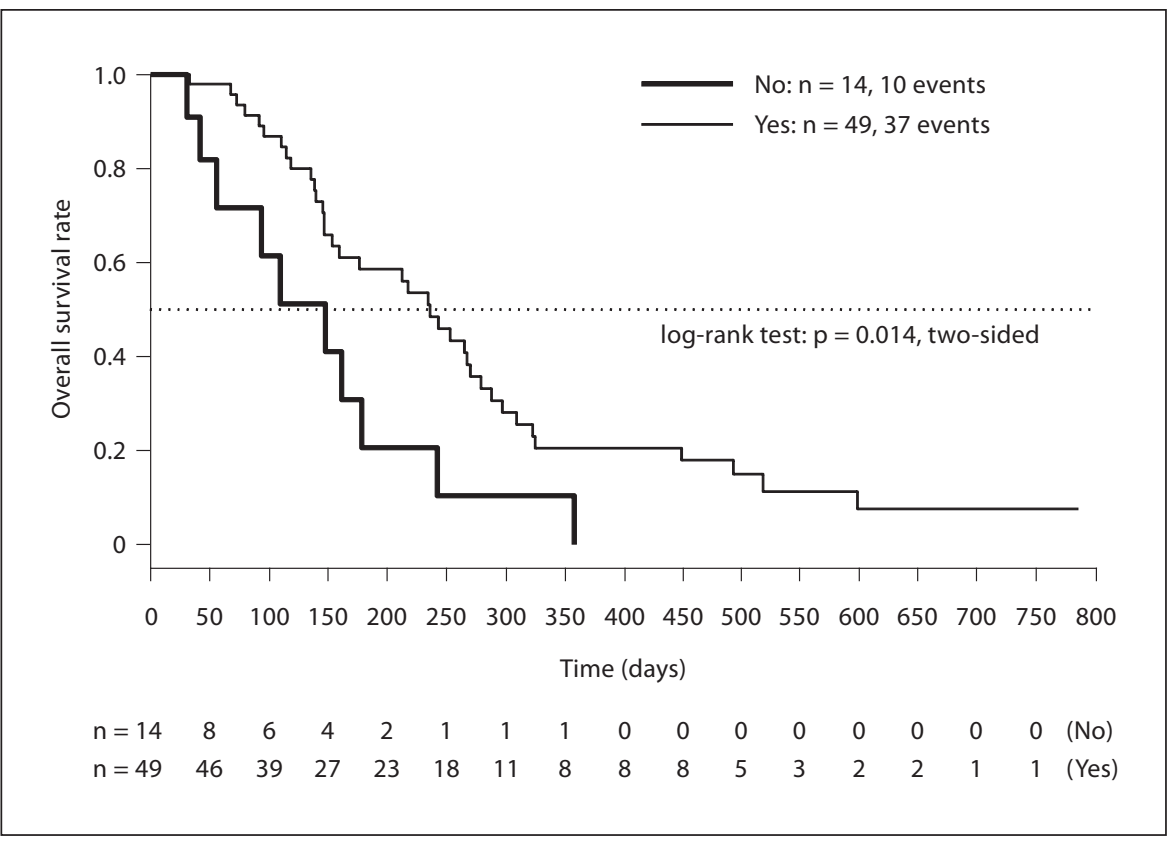

Fig. 3. Overall survival depending on rash.

cetuximab does not add any valuable activity to chemotherapy with gemcitabine or a combination of gemcitabine and cisplatin/oxaliplatin $[9,11,12]$.

Cascinu et al. [11] reported data of a phase II trial in which patients with advanced pancreatic cancer were randomly assigned to treatment with cetuximab plus gemcitabine and cisplatin or chemotherapy alone. Sixty-one of $84(73 \%)$ patients had metastatic disease. Seven of 40 (17.5\%) patients had an objective response rate in the cetuximab group and 5/41 (12.2\%) in the noncetuximab arm. No significant differences between the groups were noted in median progression-free survival or in median overall survival. Median progression-free survival was 3.4 months in the cetuximab group and 4.2 months in the noncetuximab group. Median overall survival was 7.5 and 7.8 months, respectively. Interestingly, toxic effects were not increased by cetuximab and at least 33/61 (54\%) patients with metastastic disease received a second-line fluorouracil-based chemotherapy. The findings of Cascinu et al. [11] are in agreement with a phase III study evaluating cetuximab in combination with gemcitabine compared to gemcitabine alone in advanced pancreatic cancer. Seven hundred and thirty-five patients were randomly enrolled in this latter trial and $78 \%$ had metastatic disease. The median survival was 6 months in the gemcitabine arm and 6.5 months in the gemcitabine plus cetuximab arm, which fails to demonstrate a clinically significant advantage of the addition of cetuximab to gemcitabine.
In our study on 64 patients with metastatic pancreatic carcinoma, the addition of cetuximab to the combination of gemcitabine and oxaliplatin was well tolerated and exhibited a high response rate $(33 \%)$. The median time to progression-free survival was 3.9 months and overall survival was 7.1 months. Again, these findings do not seem to be superior to the results achieved in the previous studies of gemcitabine and oxaliplatin alone.

What are the reasons for inactivity of cetuximab in pancreatic cancer and are there possibly predictive factors for response? No clinical studies evaluating efficacy of cetuximab molecular characterization including EGFR mutation have been reported so far.

Seventy to $100 \%$ of ductal adenocarcinoma exhibit KRAS mutations and all mutations were located in codon $12[13,14]$. Differences were found between the type of mutations [14, 15]. Immervoll et al. [16] screened a series of 43 formalin-fixed paraffin-embedded ductal adenocarcinoma of the pancreas. When DNA was extracted from whole tissue sections, KRAS codon 12 mutations were detected in $67 \%$ of the tumors. When cancerous ducts were isolated by laser-assisted microdissection, 91\% were positive for KRAS mutations. In the present study we performed manual microdissections and obtained a tumor cell population of $70 \%$ in every case. However, in many cases mutation analyses were done on a limited number of cells. The mutation rate in the present study was on a lower limit compared to previous studies. 
There was a trend that survival after diagnosis varied according to the KRAS mutation subtype. Patients with the Gly12Val mutation survived much longer (23.5 months) than patients with Gly12Asp mutations (9.5 months). Lee et al. [17] analyzed EGFR mutation by DNA sequencing of exon 18-21 in the tyrosine kinase domain in 65 pancreatic cancer patients. Thirty-two (49\%) of the 65 pancreatic adenocarcinomas examined harbored a point mutation in the KRAS gene. All KRAS mutations occurred at codon 12 . The most frequently observed point mutation was Gly12Val. The presence of KRAS point mutations at codon 12 adversely influenced median survival time (9.1 vs. 13.4 months, KRAS mutation (+ vs. - ), $\mathrm{p}=0.03$ ).

To our knowledge, this is the first study assessing the relationship between KRAS mutations and response or survival in patients with metastatic pancreatic cancer treated with cetuximab plus chemotherapy. Overall survival was longer in wild-type patients compared to patients with KRAS mutations (263 vs. 162 days). The difference did not reach statistical significance probably due to small sample size.

This is supported by recently reported preliminary data of the German AIO group on the role of KRAS mutation in erlotinib-treated patients with advanced pancreatic cancer [18]. Within this prospective multicenter phase III trial 281 patients were randomly assigned to first-line treatment with either capecitabine plus erlotinib or gemcitabine plus erlotinib. Tissue samples were available from 204 patients. 123 tumors (70\%) harbored a somatic KRAS mutation. Patients with KRAS wild type had a longer overall survival (wild type: 8.0 months vs. mutation: 6.6 months, HR 1.62, $\mathrm{p}=0.011$ ). On the other hand, in a molecular subset analysis of patients from the NCIC CTG PA.3, the EGFR gene copy number and KRAS mutation status were not identified as markers predictive of a survival benefit from the combination of erlotinib with gemcitabine for the first-line treatment of advanced pancreatic cancer [19].
Taken together, our results and others reported recently in the literature provide evidence that KRAS mutation in codon 12 is possibly associated with reduced survival compared to KRAS wild type. KRAS mutation could be a predictive marker in patients with advanced pancreatic cancer analogous to colorectal cancer patients treated with cetuximab. As our study was a single-arm trial with no cetuximab-free control arm, it could be possible that KRAS is just a prognostic marker and not predictive of cetuximab efficacy in pancreatic cancer. The primary limitation of the current study was as already mentioned above the low number of samples suitable for molecular analysis. Small sample size and potential selection bias make it difficult to draw firm conclusions.

Therefore, the role of EGFR targeting therapy in patients with KRAS wild-type pancreatic cancer warrants further investigation in larger trials to exclude an epiphenomenon and confirm efficacy.

A further analysis of our clinical phase II trial showed that the presence of rash was significantly correlated with overall survival, consistent with the results of Xiong et al. [8]. A similar relationship between rash and clinical outcome could be demonstrated in a large phase III study for patients with advanced pancreatic cancer treated with erlotinib [6]. The reasons for the development of a rash are not fully understood. Possible explanations include differences in drug exposure, integrity of the immune system or EGFR polymorphisms [20].

These findings support the vision of a more individualized cancer treatment.

\section{Acknowledgment}

Molecular analysis was supported by Merck Serono GmbH, Darmstadt, Germany.

\section{References}

1 Burris HA, Moore MJ, Andersen J, Green MR, Rothenberg ML, Modiano MR, Cripps MC, Portenoy RK, Storniolo AM, Tarassoff P, Nelson R, Dorr FA, Stephens CD, Von Hoff DD: Improvements in survival and clinical benefit with gemcitabine as first-line therapy for patients with advanced pancreas cancer: a randomized trial. J Clin Oncol 1997;15: 2403-2413.

-2 Heinemann V, Boeck S, Hinke A, Labianca $R$, Louvet C: Meta-analysis of randomized trials: evaluation of benefit from gemicta-

Cetuximab, Gemcitabine and Oxaliplatin in Pancreatic Cancer bine-based combination chemotherapy applied in advanced pancreatic cancer. BMC Cancer 2008;8:82.

3 Colucci G, Labianca R, Di Costanzo F, Gebbia V, Cartenì G, Massidda B, Dapretto E, Manzione L, Piazza E, Sannicolò M, Ciaparrone M, Cavanna L, Giuliani F, Maiello E, Testa A, Pederzoli P, Falconi M, Gallo C, Di Maio M, Perrone F, Gruppo Oncologico Italia Meridionale (GOIM), Gruppo Italiano per lo Studio dei Carcinomi dell'Apparato Digerente (GISCAD), Gruppo Oncologico
Italiano di Ricerca Clinica (GOIRC): Randomized phase III trial of gemcitabine plus cisplatin compared with single-agent gemcitabine as first-line treatment of patients with advanced pancreatic cancer: the GIP-1 study J Clin Oncol 2010;10:1645-1651.

-4 Fjällskog ML, Lejonklou MH, Oberg KE, Eriksson BK, Janson ET: Expression of molecular targets for tyrosine kinase receptor antagonists in malignant endocrine pancreatic tumors. Clin Cancer Res 2003;9:14691473 . 
5 Yamanaka Y, Friess H, Kobrin MS, Buchler M, Berger HG, Korc M: Coexpression of epidermal growth factor receptor and ligands in human pancreatic cancer is associated with enhanced tumor aggressiveness. Anticancer Res 1993;13:565-569.

6 Moore MJ, Goldstein D, Hamm J, Figer A, Hecht JR, Gallinger S, Au HJ, Murawa P, Walde D, Wolff RA, Campos D, Lim R, Ding K, Clark G, Voskoglou-Nomikos T, Ptasynski M, Parulekar W, National Cancer Institute of Canada Clinical Trials Group: Erlotinib plus gemcitabine compared with gemcitabine alone in patients with advanced pancreatic cancer: a phase III trial of the National Cancer Institute of Canada Clinical Trials Group. J Clin Oncol 2007;25:19601966.

7 Bruns CJ, Harbison MT, Davis DW, Portera CA, Tsan R, Mc Conkey DJ, Evans DB, Abbruzzese JL, Hicklin DJ, Radinsky R: Epidermal growth factor receptor blockade with C225 plus gemcitabine results in regression of human pancreatic carcinoma growing orthotopically in nude mice by antiangiogenic mechanisms. Clin Cancer Res 2000;6:19361948.

8 Xiong $\mathrm{HQ}$, Rosenberg A, LoBuglio A, Schmidt W, Wolff RA, Deutsch J, Needle M, Abbruzzese JL: Cetuximab, a monoclonal antibody targeting the epidermal growth factor receptor, in combination with gemcitabine for advanced pancreatic cancer: a multicenter phase II trial. J Clin Oncol 2004; 22:2610-2616.

-9 Kullmann F, Hollerbach S, Dollinger MM, Harder J, Fuchs M, Messmann H, Trojan J, Gäbele E, Hinke A, Hollerbach C, Endlicher E: Cetuximab plus gemcitabine/oxaliplatin (GEMOXCET) in 1st line metastatic pancreatic cancer - a multicenter phase II study. $\mathrm{Br}$ J Cancer 2009;100:1032-1036.
10 Lurkin I, Stoehr R, Hurst CD, van Tilborg AAG, Knowles MA, Hartmann A, Zwarthoff EC: Two multiplex assays that simultaneously identify 22 possible mutation sites in the KRAS, BRAF, NRAS and PIK3CA genes. PLosONE 2010;5:e8802.

11 Cascinu S, Berardi R, Labianca R, Siena S, Falcone A, Aitini E, Barni S, Di Costanzo F, Dapretto E, Tonini G, Pierantoni C, Artale S, Rota S, Floriani I, Scartozzi M, Zaniboni A Italian Group for the Study of Digestive Tract Cancer (GISCAD): Cetuximab plus gemcitabine and cisplatin compared with gemcitabine and cisplatin alone in patients with advanced pancreatic cancer: a randomised, multicentre, phase II trial. Lancet Oncol 2008;9:3944

12 Philip PA, Benedetti J, Corless CL, Wong R, O’Reilly EM, Flynn PJ, Rowland KM, Atkins JN, Mirtsching BC, Rivkin SE, Khorana AA, Goldman B, Fenoglio-Preiser CM, Abbruzzese JL, Blanke CD: Phase III study comparing gemcitabine plus cetuximab versus gemcitabine in patients with advanced pancreatic adenocarcinoma: Southwest Oncology Group-Directed Intergroup Trial S0205. J Clin Oncol 2010;28:3605-3610.

13 Almoguera C, Shibata D, Forrester K, Martin J, Arnheim N, Perucho M: Most human carcinomas of the exocrine pancreas contain mutant c-K-ras genes. Cell 1988;53:549-554.

14 Smit VT, Boot AJ, Smits AM, Fleuren GJ, Cornelisse CJ, Bos JL: K-ras codon 12 mutations occur very frequently in pancreatic adenocarcinomas. Nuclei Acids Res 1988;16: 7773-7782.
15 Grünewald K, Lyons J, Fröhlich A, Feichtinger $\mathrm{H}$, Weger RA, Schwab G, Janssen JWG, Bartram CH: High frequency of Ki-ras codon 12 mutations in pancreatic adenocarcinoma. Int J Cancer 1989;43:1037-1041.

$>16$ Immervoll H, Hoem D, Kugarajh K, Steine SJ, Molven A: Molecular analysis of the EGFR-RAS-RAF pathway in pancreatic ductal adenocarcinomas: lack of mutations in the BRAF and EGFR genes. Virchows Arch 2006;448:788-796.

17 Lee J, Jang KT, Ki CS, Lim T, Park YS, Lim HY, Choi DW, Kang WK, Park K, Park JO: Impact of epidermal growth factor receptor (EGFR) kinase mutations, EGFR gene amplifications, and KRAS mutations on survival of pancreatic adenocarcinoma. Cancer 2007; 109:1561-1569.

18 Boeck SH, Vehling-Kaiser U, Waldschmidt D, Kettner E, Märten A, Winkelmann C, Klein S, Kojouharoff G, Jung A, Heinemann $\mathrm{V}$ : Gemcitabine plus erlotinib (GE) followed by capecitabine $(\mathrm{C})$ versus capecitabine plus erlotinib (CE) followed by gemcitabine $(\mathrm{G})$ in advanced pancreatic cancer (APC): a randomized, cross-over phase III trial of the Arbeitsgemeinschaft Internistische Onkologie (AIO) (abstract LBA4011). J Clin Oncol 2010; 28(suppl):18s

19 da Cunha Santos G, Dhani N, Tu D, Chin K, Ludkovski O, Kamel-Reid S, Squire J, Parulekar W, Moore MJ, Tsao MS: Molecular predictors of outcome in a phase 3 study of gemcitabine and erlotinib therapy in patients with advanced pancreatic cancer. Cancer 2010;116:5599-5607.

20 Wacker B, Nagrani T, Weinberg J, Witt K, Clark G, Cagnoni PJ: Correlation between development of rash and efficacy in patients treated with epidermal growth factor receptor tyrosine kinase inhibitor erlotinib in two large phase III studies. Clin Cancer Res 2007; 13:3913-3921. 